\title{
AN ANALYTICAL AND NUMERICAL APPROACH TO A BILATERAL CONTACT PROBLEM WITH NONMONOTONE FRICTION
}

\author{
Mikä̈l BARbOTEU *, Krzysztof BARTOSZ**, Piotr KALITA** \\ * Laboratory of Applied Mathematics and Physics (LAMPS) \\ University of Perpignan, 52 Avenue Paul Alduy, 66860 Perpignan, France \\ e-mail: barboteu@univ-perp.fr \\ ** Institute of Computer Science, Faculty of Mathematics and Computer Science \\ Jagiellonian University, ul. Łojasiewicza 6, 30-348 Kraków, Poland \\ e-mail: \{bartosz,kalita\}@ii.uj.edu.pl
}

\begin{abstract}
We consider a mathematical model which describes the contact between a linearly elastic body and an obstacle, the so-called foundation. The process is static and the contact is bilateral, i.e., there is no loss of contact. The friction is modeled with a nonmotonone law. The purpose of this work is to provide an error estimate for the Galerkin method as well as to present and compare two numerical methods for solving the resulting nonsmooth and nonconvex frictional contact problem. The first approach is based on the nonconvex proximal bundle method, whereas the second one deals with the approximation of a nonconvex problem by a sequence of nonsmooth convex programming problems. Some numerical experiments are realized to compare the two numerical approaches.
\end{abstract}

Keywords: linearly elastic material, bilateral contact, nonmonotone friction law, hemivariational inequality, finite element method, error estimate, nonconvex proximal bundle method, quasi-augmented Lagrangian method, Newton method.

\section{Introduction}

This paper deals with numerical solution for frictional contact problems governed by nonmonotone friction laws. Two independent approaches inspired by the finite element method are applied and numerical results are presented for a simple two-dimensional model problem. The first approach is based on minimization of the energy functional by means of the Proximal Bundle Method (PBM) (see the works of Mäkelä $(1990 ; 2001)$ for a survey of bundle methods for nonsmooth optimization), while the second one relies on approximation of a nonconvex problem by a sequence of convex ones. The main theoretical result of the paper is Theorem 3, which provides the error estimate between the Galerkin solution and the exact one. This result is stronger than those previously obtained in the literature. It provides the strong convergence of subsequence of Galerkin solutions (see Haslinger et al., 1999, Theorem 3.12) and it is a generalization of the classical Cea lemma. The estimate is possible due to the one sided Lipschitz condition imposed on the friction bound, which is the natural assumption in frictional problems. Analogous results for the convex case are available in the works of Hild and Renard (2007) as well as Han and Sofonea (2002).

In one approach to numerical approximation of the solution based on the PBM, the Galerkin problem is converted into the form of minimization of a not necessarily convex energy functional. In each iteration step a piecewise linear approximation of the objective functional is constructed and it is regularized by adding a quadratic term. Bundle methods have already been used to solve the elastic contact problem with friction and a normal response given by a nonmonotone law by Miettinen (1995), while the delamination problem for laminated composite with nonmonotone adhesive force between laminae is solved with bundle methods by Mäkelä et al. (1999). The review of these results can be found in the monograph of Haslinger et al. (1999).

Another approach to the numerical solution of a nonconvex frictional problem consists in solving a sequence of auxiliary convex problems which approximate the original one. Each convex problem is solved by a numerical strategy based on a quasi-Lagrangean formulation combined with a Newton 
method. The approach based on the sequence of convex problems was used to solve the contact problem with nonmonotone softening behavior of the binding material in the normal direction by Tzaferopoulos et al. (1995), while the elastic contact problem with nonmonotone friction was solved by Mistakidis and Panagiotopulos $(1997 ; 1998)$. To the best of our knowledge, the literature concerning the numerical solution of nonmonotone friction problems is very limited. The bundle Newton approach was used for unilateral problems by Baniotopoulos et al. (2005).

The paper is organized as follows. In Section 2 we introduce some notations and preliminaries. In Section 3 we present the frictional contact problem together with its variational formulation. Existence and uniqueness results are also briefly presented. Section 4 is devoted to the presentation of the Galerkin problem which is the basis for the numerical schemes used to approximate the solution. The theorem on the error estimate is provided. In Section 5 we present the nonconvex PBM for the solution of the bilateral contact problem with nonmonotone friction. Section 6 concerns the solution of the nonsmooth and nonconvex problem by using a numerical strategy based on a sequence of convex programming problems. In the last section we present some numerical examples to compare the two preceding numerical methods for the solution of bilateral contact problems with a nonmonotone friction law.

\section{Notation and preliminaries}

In this section we present the notation we shall use along with some preliminary material. For further details we refer the reader to the works of Duvaut and Lions (1976), Ionescu and Sofonea (1993), as well as Panagiotopoulos (1985)

We denote by $\mathbb{S}^{d}$ the space of second order symmetric tensors on $\mathbb{R}^{d}$ ( $d \leq 3$ in applications), while "." and $\mid$. | will represent the inner product and the Euclidean norm on $\mathbb{S}^{d}$ and $\mathbb{R}^{d}$, respectively, i.e.,

$$
\begin{array}{lll}
\boldsymbol{u} \cdot \boldsymbol{v}=u_{i} v_{i}, & |\boldsymbol{v}|=(\boldsymbol{v} \cdot \boldsymbol{v})^{\frac{1}{2}}, & \forall \boldsymbol{u}, \boldsymbol{v} \in \mathbb{R}^{d}, \\
\boldsymbol{\sigma} \cdot \boldsymbol{\varepsilon}=\sigma_{i j} \varepsilon_{i j}, & |\varepsilon|=(\varepsilon \cdot \boldsymbol{\varepsilon})^{\frac{1}{2}}, & \forall \boldsymbol{\sigma}, \boldsymbol{\varepsilon} \in \mathbb{S}^{d} .
\end{array}
$$

Here and below the indices $i$ and $j$ run between 1 and $d$, and the summation convention over repeated indices is adopted.

Let $\Omega \subset \mathbb{R}^{d}$ be a bounded domain with a Lipschitz boundary $\Gamma$, and let $\boldsymbol{n}$ denote the unit outer normal on $\Gamma$ and $\tau$ the associated tangent vector. We shall use the notation

$$
H=L^{2}(\Omega)^{d}=\left\{\boldsymbol{u}=\left(u_{i}\right) \mid u_{i} \in L^{2}(\Omega)\right\},
$$

$$
\begin{aligned}
Q & =\left\{\boldsymbol{\sigma}=\left(\sigma_{i j}\right) \mid \sigma_{i j}=\sigma_{j i} \in L^{2}(\Omega)\right\}, \\
H_{1} & =\left\{\boldsymbol{u}=\left(u_{i}\right) \mid \boldsymbol{\varepsilon}(\boldsymbol{u}) \in Q\right\}, \\
Q_{1} & =\{\boldsymbol{\sigma} \in Q \mid \operatorname{Div} \boldsymbol{\sigma} \in H\} .
\end{aligned}
$$

Here $\varepsilon: H_{1} \longrightarrow Q$ and Div $: Q_{1} \longrightarrow H$ are the $d e$ formation and divergence operators, respectively, defined by

$$
\boldsymbol{\varepsilon}(\boldsymbol{u})=\left(\varepsilon_{i j}(\boldsymbol{u})\right), \quad \varepsilon_{i j}(\boldsymbol{u})=\frac{1}{2}\left(u_{i, j}+u_{j, i}\right),
$$

$\operatorname{Div} \boldsymbol{\sigma}=\left(\sigma_{i j, j}\right)$,

where the index that follows a comma indicates a partial derivative with respect to the corresponding component of the independent variable. The spaces $H, Q, H_{1}$ and $Q_{1}$ are real Hilbert spaces endowed with the canonical inner products given by

$$
\begin{aligned}
(\boldsymbol{u}, \boldsymbol{v})_{H} & =\int_{\Omega} u_{i} v_{i} \mathrm{~d} x \\
(\boldsymbol{\sigma}, \varepsilon)_{Q} & =\int_{\Omega} \sigma_{i j} \varepsilon_{i j} \mathrm{~d} x \\
(\boldsymbol{u}, \boldsymbol{v})_{H_{1}} & =(\boldsymbol{u}, \boldsymbol{v})_{H}+(\boldsymbol{\varepsilon}(\boldsymbol{u}), \boldsymbol{\varepsilon}(\boldsymbol{v}))_{Q} \\
(\boldsymbol{\sigma}, \varepsilon)_{Q_{1}} & =(\boldsymbol{\sigma}, \varepsilon)_{Q}+(\operatorname{Div} \boldsymbol{\sigma}, \operatorname{Div} \varepsilon)_{H} .
\end{aligned}
$$

The associated norms on these spaces are further denoted by $\|\cdot\|_{H},\|\cdot\|_{Q},\|\cdot\|_{H_{1}}$ and $\|\cdot\|_{Q_{1}}$, respectively.

Let $H_{\Gamma}=H^{1 / 2}(\Gamma)^{d}$ and let $\gamma: H_{1} \longrightarrow H_{\Gamma}$ be the trace map. For every element $\boldsymbol{v} \in H_{1}$ we still write $\boldsymbol{v}$ to denote the trace $\gamma \boldsymbol{v}$ of $\boldsymbol{v}$ on $\Gamma$, and we denote by $v_{n}$ and $\boldsymbol{v}_{\tau}$ the normal and tangential components of $\boldsymbol{v}$ on the boundary $\Gamma$ given by

$$
v_{n}=\boldsymbol{v} \cdot \boldsymbol{n}, \quad \boldsymbol{v}_{\tau}=\boldsymbol{v}-v_{n} \boldsymbol{n} .
$$

Let $H_{\Gamma}^{*}$ be the dual of $H_{\Gamma}$ and let $(\cdot, \cdot)$ denote the duality pairing between $H_{\Gamma}^{*}$ and $H_{\Gamma}$. For every $\boldsymbol{\sigma} \in Q_{1}$ there exists an element $\boldsymbol{\sigma} \boldsymbol{n} \in H_{\Gamma}^{*}$ such that

$$
(\boldsymbol{\sigma}, \boldsymbol{\varepsilon}(\boldsymbol{v}))_{Q}+(\operatorname{Div} \boldsymbol{\sigma}, \boldsymbol{v})_{H}=(\boldsymbol{\sigma} \boldsymbol{n}, \gamma \boldsymbol{v}), \quad \forall \boldsymbol{v} \in H_{1}
$$

Moreover, if $\boldsymbol{\sigma}$ is a smooth (say $C^{1}$ ) function, then

$$
(\boldsymbol{\sigma n}, \gamma \boldsymbol{v})=\int_{\Gamma} \boldsymbol{\sigma n} \cdot \boldsymbol{v} \mathrm{d} \Gamma, \quad \forall \boldsymbol{v} \in H_{1}
$$

We also denote by $\sigma_{n}$ and $\boldsymbol{\sigma}_{\tau}$ the normal and tangential traces of $\sigma$ and we recall that, when $\sigma$ is smooth enough, then

$$
\sigma_{n}=(\boldsymbol{\sigma n}) \cdot \boldsymbol{n}, \quad \boldsymbol{\sigma}_{\tau}=\boldsymbol{\sigma} \boldsymbol{n}-\sigma_{n} \boldsymbol{n} .
$$

Finally, we recall definitions of the generalized derivative and gradient (see Clarke, 1983). Let $X$ be a reflexive Banach space and $X^{*}$ its dual. The Clarke generalized directional derivative of a locally Lipschitz 
function $h: X \rightarrow \mathbb{R}$ at the point $x \in X$ in the direction $v \in X$, denoted by $h^{0}(x ; v)$, is defined by

$$
h^{0}(x ; v)=\limsup _{y \rightarrow x, \lambda \downarrow 0} \frac{h(y+\lambda v)-h(y)}{\lambda} .
$$

The Clarke subdifferential of $h$ at $x$ denoted by $\partial h(x)$ is a subset of $X^{*}$ given by $\partial h(x)=\left\{\zeta \in X^{*}: h^{0}(x ; v) \geq\right.$ $\langle\zeta, v\rangle_{X^{*} \times X}$ for all $\left.v \in X\right\}$.

\section{Mechanical problem and variational formulation}

In this section we describe the model for the nonmonotone frictional bilateral contact, present the variational formulation well suited for the numerical treatment used in the sequel, and finally recall an existence and uniqueness result.

The physical setting is as follows. A linearly elastic body occupies an open bounded connected set $\Omega \subset \mathbb{R}^{d}$ ( $d \leq 3$ in applications) with a Lipschitz boundary $\Gamma$ that is partitioned into three disjoint parts $\bar{\Gamma}_{1}, \bar{\Gamma}_{2}$ and $\bar{\Gamma}_{3}$ with $\Gamma_{1}, \Gamma_{2}$ and $\Gamma_{3}$ being relatively open, and meas $\left(\Gamma_{1}\right)>0$. The body is clamped on $\Gamma_{1}$ and thus the displacement field vanishes there. A volume force of density $\boldsymbol{f}_{0}$ acts in $\Omega$ and a surface traction of density $f_{2}$ acts on $\Gamma_{2}$. The body is in frictional contact with an obstacle on $\Gamma_{3}$. We assume that there is no loss of contact during the process, i.e., the contact is bilateral. Thus, the normal displacement $u_{n}$ vanishes on $\Gamma_{3}$. We model the friction by a nonmonotone friction law. The material is linearly elastic and the process is assumed to be static.

In the study of the frictional contact problem we need the following assumptions on its data:

- $H(\mathcal{E})$ : the elasticity operator $\mathcal{E}: \Omega \times \mathbb{S}^{d} \rightarrow \mathbb{S}^{d}$ is a bounded symmetric positive definite fourth order tensor, i.e.,

$$
\left\{\begin{array}{l}
\text { (a) } \mathcal{E}_{i j k l} \in L^{\infty}(\Omega), 1 \leq i, j, k, l \leq d ; \\
\text { (b) } \mathcal{E} \boldsymbol{\sigma} \cdot \boldsymbol{\tau}=\boldsymbol{\sigma} \cdot \mathcal{E} \boldsymbol{\tau}, \forall \boldsymbol{\sigma}, \boldsymbol{\tau} \in \mathbb{S}^{d} \text {, a.e. in } \Omega \text {; } \\
\text { (c) } \mathcal{E} \boldsymbol{\tau} \cdot \boldsymbol{\tau} \geq m|\boldsymbol{\tau}|^{2}, \forall \boldsymbol{\tau} \in \mathbb{S}^{d} \text {, a.e. in } \Omega \\
\text { with } m>0 .
\end{array}\right.
$$

- $H(f)$ : the force and the traction densities satisfy

$$
\boldsymbol{f}_{0} \in L^{2}(\Omega)^{d}, \quad \boldsymbol{f}_{2} \in L^{2}\left(\Gamma_{2}\right)^{d} \text {. }
$$

- $H(\mu)$ : the friction bound satisfies

$$
\left\{\begin{array}{l}
\text { (a) } \mu:[0, \infty) \rightarrow \mathbb{R} \text { is measurable; } \\
\text { (b) } \mu(0)=\lim _{x \rightarrow 0^{+}} \mu(x)>0 ; \\
\text { (c) }-c_{2} \leq \mu(t) \leq c_{1}(1+t), \forall t \geq 0, \\
\text { with } c_{1}, c_{2}>0 ; \\
\text { (d) } \mu\left(t_{1}\right)-\mu\left(t_{2}\right) \geq-\lambda\left(t_{1}-t_{2}\right), \forall t_{1}>t_{2} \geq 0, \\
\text { with } \lambda>0 .
\end{array}\right.
$$

Remark 1. The hypothesis $H(\mu)$ (c) implies that

$$
|\mu(t)| \leq c(1+t), \forall t \geq 0, \quad \text { with } c>0,
$$

and

$$
\mu(t) \geq-d\left(1+\frac{1}{t}\right), \forall t>0, \quad \text { with } d>0,
$$

Remark 2. In a particular case, since $\mu$ corresponds to the coefficient of friction, it is nonnegative, so the lower bound in (c) obviously holds. The condition (d) is the so-called one-side Lipschitz condition, which allows the function to decrease with a rate not faster than $\lambda$.

The classical formulation of the mechanical problem is the following.

Problem 1. $\left(\mathcal{P}_{M}\right)$ Find a displacement field $\boldsymbol{u}: \Omega \rightarrow \mathbb{R}^{d}$ and a stress field $\sigma: \Omega \rightarrow \mathbb{S}^{d}$ such that

$$
\begin{aligned}
& \operatorname{Div} \boldsymbol{\sigma}+\boldsymbol{f}_{0}=\mathbf{0} \quad \text { in } \Omega \text {, } \\
& \boldsymbol{\sigma}=\mathcal{E} \varepsilon(\boldsymbol{u}) \text { in } \Omega, \\
& \boldsymbol{u}=\mathbf{0} \text { on } \Gamma_{1} \text {, } \\
& \boldsymbol{\sigma} \boldsymbol{n}=\boldsymbol{f}_{2} \quad \text { on } \Gamma_{2} \text {, } \\
& u_{n}=0 \quad \text { on } \Gamma_{3} \text {, }
\end{aligned}
$$

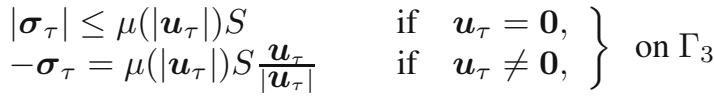

In (13) $\mu\left(\left|\boldsymbol{u}_{\tau}\right|\right) S$ represents the magnitude of the limiting friction traction at which slip begins. Here, $S \geq 0$ is a given value. The friction bound and, more precisely, the friction coefficient $\mu$ depend on the tangential displacement $\left|\boldsymbol{u}_{\tau}\right|$. The strict inequality in (13) holds in the stick zone and the equality holds in the slip zone. This physical model of slip-dependent friction was introduced by Rabinowicz (1951) for the geophysical context of earthquake modeling, and was also studied by Ionescu and Paumier (1996), Ionescu and Nguyen (2002), Ionescu et al. (2003), Shillor et al. (2004), as well as Migórski and Ochal (2005). Due to the basic properties of the Clarke subdifferential (cf. Clarke, 1983), the right-hand side of the friction conditions (13) can be written as a subdifferential of a locally Lipshitz, 
possibly nonconvex superpotential $j_{s}$ which depends on the tangential displacement $\boldsymbol{u}_{\tau}$. In fact, if the function $j_{s}: \mathbb{R}^{d} \rightarrow \mathbb{R}$ is defined by

$$
j_{s}(\boldsymbol{\xi})=S \int_{0}^{|\boldsymbol{\xi}|} \mu(t) \mathrm{d} t
$$

then we can prove that under assumptions $H(\mu)(\mathrm{a})-(\mathrm{c})$ the conditions (13) are equivalent to the following subdifferential inclusion:

$$
-\boldsymbol{\sigma}_{\tau} \in \partial j_{s}\left(\boldsymbol{u}_{\tau}\right) \text { on } \Gamma_{3},
$$

where $\partial j_{s}(\boldsymbol{\xi})$ denotes the Clarke subdifferential of $j_{s}$. By means of basic calculations, one can easily prove the following lemma on the properties of the function $j_{s}$,

Lemma 1. If the assumptions $H(\mu)(a)-(c)$ hold, then the function $j_{s}$ defined by (14) has the following properties:

- $H\left(j_{s}\right)$ :

$$
\left\{\begin{array}{l}
\text { (a) } j_{s} \text { is locally Lipschitz; } \\
\text { (b) }|\boldsymbol{\eta}| \leq S c(1+|\boldsymbol{\xi}|), \forall \boldsymbol{\xi} \in \mathbb{R}^{d}, \boldsymbol{\eta} \in \partial j_{s}(\boldsymbol{\xi}) \\
\text { (c) } j_{s}^{0}(\boldsymbol{\xi} ;-\boldsymbol{\xi}) \leq S d(1+|\boldsymbol{\xi}|), \forall \boldsymbol{\xi} \in \mathbb{R}^{d}
\end{array}\right.
$$

Furthermore if the assumption $H(\mu)(d)$ holds, then we have

$$
\begin{gathered}
(d)\left(\boldsymbol{\eta}_{1}-\boldsymbol{\eta}_{2}\right) \cdot\left(\boldsymbol{\xi}_{1}-\boldsymbol{\xi}_{2}\right) \geq-S \lambda\left|\boldsymbol{\xi}_{1}-\boldsymbol{\xi}_{2}\right|^{2}, \\
\forall \boldsymbol{\xi}_{1}, \boldsymbol{\xi}_{2} \in \mathbb{R}^{d}, \boldsymbol{\eta}_{i} \in \partial j_{s}\left(\boldsymbol{\xi}_{i}\right), i=1,2 .
\end{gathered}
$$

Corollary 1. If the functional $j_{s}: \mathbb{R}^{d} \rightarrow \mathbb{R}$ satisfies $H\left(j_{s}\right)(a)-(c)$, then

$$
j_{s}^{0}(\boldsymbol{\xi} ;-\boldsymbol{\xi}) \leq S(c+d)|\boldsymbol{\xi}|, \quad \forall \boldsymbol{\xi} \in \mathbb{R}^{d} .
$$

Proof. If $|\boldsymbol{\xi}| \leq d / c$, then we have

$$
\begin{aligned}
j_{s}^{0}(\boldsymbol{\xi} ;-\boldsymbol{\xi})= & \max _{\boldsymbol{v} \in \partial j_{s}(\boldsymbol{\xi})} \boldsymbol{v} \cdot(-\boldsymbol{\xi}) \\
& \leq \max _{\boldsymbol{v} \in \partial j_{s}(\boldsymbol{\xi})}|\boldsymbol{v} \| \boldsymbol{\xi}| \\
& \leq S c(1+|\boldsymbol{\xi}|)|\boldsymbol{\xi}| \leq S(c+d)|\boldsymbol{\xi}|
\end{aligned}
$$

If $|\boldsymbol{\xi}| \geq d / c$, then we have $1 \leq c / d|\boldsymbol{\xi}|$, so

$$
j_{s}^{0}(\boldsymbol{\xi} ;-\boldsymbol{\xi}) \leq S d(1+|\boldsymbol{\xi}|) \leq S(c+d)|\boldsymbol{\xi}|
$$

We now turn to the variational formulation for the mechanical problem $\mathcal{P}_{M}$. To this end, we introduce the closed subspace of $H_{1}$ defined by

$$
V=\left\{\boldsymbol{v} \in H_{1} \mid \boldsymbol{v}=\mathbf{0} \text { on } \Gamma_{1}, v_{n}=0 \text { on } \Gamma_{3}\right\} .
$$

Since meas $\left(\Gamma_{1}\right)>0$, Korn's inequality holds, and thus there exists $C_{K}>0$ which depends only on $\Omega$ and $\Gamma_{1}$ such that

$$
\|\varepsilon(\boldsymbol{v})\|_{Q} \geq C_{K}\|\boldsymbol{v}\|_{H_{1}} \quad \forall \boldsymbol{v} \in V .
$$

A proof of Korn's inequality may be found in the work of Nečas and Hlavaček (1981, p. 79). On $V$, we consider the inner product given by

$$
(\boldsymbol{u}, \boldsymbol{v})_{V}=(\varepsilon(\boldsymbol{u}), \boldsymbol{\varepsilon}(\boldsymbol{v}))_{Q}, \quad \forall \boldsymbol{u}, \boldsymbol{v} \in V .
$$

Let $\|\cdot\|$ be the associated norm, i.e.,

$$
\|\boldsymbol{v}\|=\|\varepsilon(\boldsymbol{v})\|_{Q}, \quad \forall \boldsymbol{v} \in V .
$$

It follows from (17) and (19) that $\|\cdot\|_{H_{1}}$ and $\|\cdot\|$ are equivalent norms on $V$ and therefore $(V,\|\cdot\|)$ is a real Hilbert space. The duality pairing between $V$ and $V^{*}$ is denoted by $\langle\cdot, \cdot\rangle$. Moreover, by the Sobolev trace theorem and 17. we have a constant $C_{0}$ depending only on the domain $\Omega, \Gamma_{1}$ and $\Gamma_{3}$ such that

$$
\|\boldsymbol{v}\|_{L^{2}\left(\Gamma_{3}\right)^{d}} \leq C_{0}\|\boldsymbol{v}\|, \quad \forall \boldsymbol{v} \in V .
$$

Next we define the operator $B: V \rightarrow V^{*}$ and the bilinear form $a: V \times V \rightarrow \mathbb{R}$ by

$$
\langle B \boldsymbol{u}, \boldsymbol{v}\rangle=a(\boldsymbol{u}, \boldsymbol{v})=(\mathcal{E} \varepsilon(\boldsymbol{u}), \boldsymbol{\varepsilon}(\boldsymbol{v}))_{Q},
$$

and the functional $J_{s}: V \rightarrow \mathbb{R}$ by

$$
J_{s}(\boldsymbol{v})=\int_{\Gamma_{3}} j_{s}\left(\boldsymbol{v}_{\tau}(x)\right) \mathrm{d} \Gamma .
$$

We also denote by $\boldsymbol{f}$ the element of $V^{*}$ given by

$$
\langle\boldsymbol{f}, \boldsymbol{v}\rangle=\int_{\Omega} \boldsymbol{f}_{0} \cdot \boldsymbol{v} \mathrm{d} x+\int_{\Gamma_{2}} \boldsymbol{f}_{2} \cdot \boldsymbol{v} \mathrm{d} \Gamma
$$

for all $v \in V$. From (6), it follows that the integrals in (23) are well defined.

Proceeding in a standard way, we obtain the following variational formulation of the frictional Problem $\mathcal{P}_{M}$.

Problem 2. $\left(\mathcal{P}_{V}^{1}\right)$ Find a displacement field $\boldsymbol{u} \in V$ and the friction density $\boldsymbol{\xi}_{\tau} \in L^{2}\left(\Gamma_{3}\right)^{d}$ such that

$$
\langle B \boldsymbol{u}-\boldsymbol{f}, \boldsymbol{v}\rangle=\int_{\Gamma_{3}} \boldsymbol{\xi}_{\tau} \cdot \boldsymbol{v}_{\tau} \mathrm{d} \Gamma, \quad \forall \boldsymbol{v} \in V
$$

with

$$
-\boldsymbol{\xi}_{\tau} \in \partial j_{s}\left(\boldsymbol{u}_{\tau}\right) \text { a.e. on } \Gamma_{3} .
$$

The above problem is called a boundary HemiVariational Inequality (HVI). Now we define an auxiliary problem.

Problem 3. $\left(\mathcal{P}_{V}^{2}\right)$ Find a displacement field $\boldsymbol{u} \in V$ such that

$$
\boldsymbol{f}-B \boldsymbol{u} \in \partial J_{s}(\boldsymbol{u}) \text { in } V^{*} .
$$


Remark 3. Basic properties of the Clarke subdifferential (see Clarke, 1983, Theorem 2.7.2) guarantee that each solution to Problem $\mathcal{P}_{V}^{2}$ also solves Problem $\mathcal{P}_{V}^{1}$.

Now we formulate the existence theorem for Problem $\mathcal{P}_{V}^{1}$.

Theorem 1. If the assumptions $H(\mathcal{E})(a)-(c), H(\mu)(a)-$ (c) and $H(f)$ hold, then Problem $\mathcal{P}_{V}^{1}$ has a solution.

Remark 4. Due to the growth condition $H\left(j_{s}\right)$ (b) we have that $\boldsymbol{\xi}_{\tau} \in L^{2}\left(\Gamma_{3}\right)^{d}$. This regularity is stronger than the typical regularity $\boldsymbol{\xi}_{\tau} \in X_{\tau}^{*}$, where $X_{\tau}=\left\{\boldsymbol{v}_{\mid \Gamma_{3}}, \boldsymbol{v} \in\right.$ $V\}$ obtained by Khenous et al. (2006a; 2006b).

Since the proof of Theorem 1 is based on the standard technique (cf. Naniewicz and Panagiotopoulos, 1995), we present only a short outline. First, we formulate the following lemmas, which can be easily proved.

Lemma 2. If the assumptions $H(\mathcal{E})(a)-(c)$ hold, then the operator $B \in \mathcal{L}\left(V, V^{*}\right)$ is symmetric $(\forall \boldsymbol{u}, \boldsymbol{v} \in$ $V\langle B \boldsymbol{u}, \boldsymbol{v}\rangle=\langle B \boldsymbol{v}, \boldsymbol{u}\rangle)$ and coercive $(\forall \boldsymbol{u} \in$ $V\langle B \boldsymbol{u}, \boldsymbol{u}\rangle \geq m\|\boldsymbol{u}\|^{2}$ with $m>0$ ).

Lemma 3. If the assumptions $H(\mu)(a)-(c)$ hold, then the functional $J_{s}$ defined by (22) satisfies the following:

$$
\left\{\begin{array}{c}
\text { (a) } J_{s} \text { is well defined and locally Lipschitz; } \\
\text { (b) }\|\boldsymbol{\eta}\|_{V^{*}} \leq C(1+\|\boldsymbol{u}\|), \forall \boldsymbol{u} \in V, \boldsymbol{\eta} \in \partial J_{s}(\boldsymbol{u}) \\
\text { with a constant } C>0 ; \\
\text { (c) } J_{s}^{0}(\boldsymbol{u} ;-\boldsymbol{u}) \leq D(1+\|\boldsymbol{u}\|), \forall \boldsymbol{u} \in V \\
\text { with a constant } D>0 .
\end{array}\right.
$$

We now turn to the proof of Theorem 1

Proof. Due to Remark 3 it is enough to prove the existence of a solution to Problem $\mathcal{P}_{V}^{2}$. To this end, we formulate it in the equivalent way: Find $v \in V$ such that

$$
T u \ni f,
$$

where $T: V \rightarrow 2^{V^{*}}$ is a multivalued operator defined by

$$
T(\cdot)=B+\partial J_{s}(\cdot) .
$$

We need to verify that the operator $T$ is coercive and pseudomonotone and exploit the surjectivity result of Brezis (see, e.g., Denkowski et al., 2003, Theorem 1.3.70). The coercivity of $T$ follows from the condition (c) of Lemma 3 and the coercivity of $B$. We observe that $\partial J_{s}$ is generalized pseudomonotone (see Clarke, 1983, Proposition 2.1.5(b); Denkowski et al., 2003, Definition 1.3.63). Furthermore, $\partial J_{s}$ has nonempty, convex and closed values (see Clarke, 1983, Proposition 2.1.2(a)) and, by the condition (b) in Lemma 3. it is bounded. By Theorem 1.3.66 of Denkowski et al. (2003), $\partial J_{s}$ is pseudomonotone. Linearity, continuity and coercivity of $B$ imply its pseudomonotonicity. Thus $T$ is pseudomonotone, which completes the proof.
Now we pass to the uniqueness result for Problem $\mathcal{P}_{V}^{1}$.

Theorem 2. If the assumptions $H(\mathcal{E})(a)-(c), H(\mu)(a)$ (d), $H(f)$ hold and

$$
m>S \lambda C_{0}^{2},
$$

then the solution to Problem $\mathcal{P}_{V}^{1}$ is unique and Problems $\mathcal{P}_{V}^{1}$ and $\mathcal{P}_{V}^{2}$ are equivalent.

Proof. Let $\left(\boldsymbol{u}^{1}, \boldsymbol{\xi}_{\tau}^{1}\right),\left(\boldsymbol{u}^{2}, \boldsymbol{\xi}_{\tau}^{2}\right)$ solve Problem $\mathcal{P}_{V}^{1}$. We consider Eqn. (24) for both solutions separately. Subtracting the equations from each other we get

$$
\left\langle B\left(\boldsymbol{u}^{1}-\boldsymbol{u}^{2}\right), \boldsymbol{v}\right\rangle=\int_{\Gamma_{3}}\left(\boldsymbol{\xi}_{\tau}^{1}-\boldsymbol{\xi}_{\tau}^{2}\right) \cdot \boldsymbol{v}_{\tau} \mathrm{d} \Gamma, \quad \forall \boldsymbol{v} \in V .
$$

Let us take $\boldsymbol{v}=\boldsymbol{u}^{1}-\boldsymbol{u}^{2}$ in (29). By the condition $H(\mathcal{E})(\mathrm{c})$, Lemma 2 and Lemma 11d) as well as (20), we obtain

$$
m\left\|\boldsymbol{u}^{1}-\boldsymbol{u}^{2}\right\|^{2} \leq S \lambda C_{0}^{2}\left\|\boldsymbol{u}^{1}-\boldsymbol{u}^{2}\right\|^{2},
$$

which implies that $\left\|\boldsymbol{u}^{1}-\boldsymbol{u}^{2}\right\|=0$ if (28) holds. From 29] it follows that $\int_{\Gamma_{3}}\left(\boldsymbol{\xi}_{\tau}^{1}-\boldsymbol{\xi}_{\tau}^{2}\right) \cdot \boldsymbol{v}_{\tau} \mathrm{d} \Gamma=0$. The equality $\boldsymbol{\xi}_{\tau}^{1}=\boldsymbol{\xi}_{\tau}^{2}$ comes from the density of the traces of the elements of $V$ in the space $X_{\tau}$.

Let now $\boldsymbol{u} \in V$ be the solution to Problem $\mathcal{P}_{V}^{1}$ and let $\sigma$ be the stress field given by (9). By a standard procedure, it can be shown that

$$
\operatorname{Div} \boldsymbol{\sigma}+\boldsymbol{f}_{0}=\mathbf{0} \text { a.e. in } \Omega .
$$

Thus, $\boldsymbol{\sigma} \in Q_{1}$. A pair of functions $(\boldsymbol{u}, \boldsymbol{\sigma})$ which satisfies (24) and (9) is called a weak solution of the bilateral contact problem with nonmonotone friction law (9)-(13). Thus Problem $\mathcal{P}_{M}$ has a unique weak solution. Note that under the assumption

$$
\boldsymbol{\sigma} \boldsymbol{n} \in L^{2}(\Gamma)^{d}
$$

it can be further shown that

$$
\boldsymbol{\sigma} \boldsymbol{n}=\boldsymbol{f}_{2} \quad \text { a.e. on } \Gamma_{2}
$$

and

$$
-\boldsymbol{\sigma}_{\tau} \in \partial j_{s}\left(\boldsymbol{u}_{\tau}\right) \quad \text { a.e. on } \Gamma_{3} \text {. }
$$

\section{Galerkin approximation and the error estimate}

In this section we present the numerical scheme for Problem $\mathcal{P}_{V}^{1}$ based on the Galerkin method as well as the estimates for the error of this scheme.

Let $V^{h}$ be a finite dimensional linear subspace of $V$ equipped with the norm of $V$, where $h>0$ denotes 
the spatial discretization parameter. In the numerical simulations presented in the next sections, $V^{h}$ consists of continuous and piecewise affine functions, that is,

$$
\begin{array}{r}
V^{h}=\left\{\boldsymbol{v}^{h} \in[C(\bar{\Omega})]^{d}: \forall T r \in \mathcal{T}^{h} \quad \boldsymbol{v}_{\left.\right|_{T r}}^{h} \in\left[P_{1}(T r)\right]^{d},\right. \\
\left.\boldsymbol{v}^{h}=\mathbf{0} \text { on } \Gamma_{1}, v_{n}^{h}=0 \text { on } \Gamma_{3}\right\},
\end{array}
$$

where $\Omega$ is assumed to be a polygonal domain, $\mathcal{T}^{h}$ denotes a finite element triangulation of $\bar{\Omega}$, and $P_{1}(T r)$ represents the space of polynomials of global degree less or equal to one in $T r$. The discrete approximation of Problem $\mathcal{P}_{V}^{1}$ takes the following form

Problem 4. $\left(\mathcal{P}_{V}^{h}\right)$ Find a displacement field $\boldsymbol{u}^{h} \in V^{h}$ and the friction density $\boldsymbol{\xi}_{\tau}^{h} \in L^{2}\left(\Gamma_{3}\right)^{d}$ such that

$$
\left\langle B \boldsymbol{u}^{h}-\boldsymbol{f}, \boldsymbol{v}^{h}\right\rangle=\int_{\Gamma_{3}} \boldsymbol{\xi}_{\tau}^{h} \cdot \boldsymbol{v}_{\tau}^{h} \mathrm{~d} \Gamma, \quad \forall \boldsymbol{v}^{h} \in V^{h}
$$

with

$$
-\boldsymbol{\xi}_{\tau}^{h} \in \partial j_{s}\left(\boldsymbol{u}_{\tau}^{h}\right) \text { a.e. on } \Gamma_{3} \text {. }
$$

The existence and uniqueness of solutions to this problem can be proved analogously to those for Problem $\mathcal{P}_{V}^{1}$. We now formulate a result which estimates the error between the solutions to Problems $\mathcal{P}_{V}^{1}$ and $\mathcal{P}_{V}^{h}$.

Theorem 3. Let $\boldsymbol{u} \in V$ and $\boldsymbol{u}^{h} \in V^{h}$ solve Problems $\mathcal{P}_{V}^{1}$ and $\mathcal{P}_{V}^{h}$, respectively. If the assumptions $H(\mathcal{E})(a)$ (c), $H(\mu)(a)-(d), H(f)$ and (28) hold, then there exists a constant $C_{1}>0$, depending only on the given data, such that

$$
\left\|\boldsymbol{u}-\boldsymbol{u}^{h}\right\| \leq C_{1} \sqrt{r},
$$

where $r=\inf \boldsymbol{v}^{h} \in V^{h}\left\|\boldsymbol{u}-\boldsymbol{v}^{h}\right\|$.

Proof. $\quad$ Let $\boldsymbol{u} \in V$ and $\boldsymbol{u}^{h} \in V^{h}$ solve $\mathcal{P}_{V}^{1}$ and $\mathcal{P}_{V}^{h}$, respectively. Let us take $\boldsymbol{\xi}_{\tau}$ and $\boldsymbol{\xi}_{\tau}^{h}$ for which the pairs $\left(\boldsymbol{u}, \boldsymbol{\xi}_{\tau}\right)$ and $\left(\boldsymbol{u}^{h}, \boldsymbol{\xi}_{\tau}^{h}\right)$ satisfy the relations (24) and (34), respectively. First we estimate the norm of the solution $\boldsymbol{u}$. To this end, we take $\boldsymbol{v}=\boldsymbol{u}$ in (24) and by Lemma 2. the definition of the Clarke subdifferential, Corollary 1 and the Hölder inequality we obtain

$$
\begin{aligned}
m\|\boldsymbol{u}\|^{2} & \leq\langle B \boldsymbol{u}, \boldsymbol{u}\rangle=\langle f, \boldsymbol{u}\rangle+\int_{\Gamma_{3}}-\boldsymbol{\xi}_{\tau} \cdot\left(-\boldsymbol{u}_{\tau}\right) \mathrm{d} \Gamma \\
& \leq\|f\|_{V^{*}}\|\boldsymbol{u}\|+S(c+d) \int_{\Gamma_{3}}\left|\boldsymbol{u}_{\tau}\right| \mathrm{d} \Gamma \\
& \leq\|\boldsymbol{u}\|\left(\|f\|_{V^{*}}+S(c+d) C_{0} \sqrt{\operatorname{meas}\left(\Gamma_{3}\right)}\right),
\end{aligned}
$$

where meas $\left(\Gamma_{3}\right)$ denotes the Lebesgue measure of the set $\Gamma_{3}$. From (36) we get

$$
\|\boldsymbol{u}\| \leq \alpha:=\frac{\|f\|_{V^{*}}+S(c+d) C_{0} \sqrt{\operatorname{meas}\left(\Gamma_{3}\right)}}{m} .
$$

Now we estimate the norm of $\boldsymbol{\xi}_{\tau}$ by means of $\|\boldsymbol{u}\|$. Integrating the inequality $H\left(j_{s}\right)$ (b) over $\Gamma_{3}$ we obtain by simple calculations

$$
\begin{aligned}
&\left\|\boldsymbol{\xi}_{\tau}\right\|_{L^{2}\left(\Gamma_{3}\right)^{d}} \\
& \leq S c \sqrt{2 \mathrm{meas}\left(\Gamma_{3}\right)}+\sqrt{2} S c C_{0}\|\boldsymbol{u}\|:=\beta+\delta\|\boldsymbol{u}\| .
\end{aligned}
$$

We can obtain an analogous estimate for $\boldsymbol{u}^{h}$ and $\boldsymbol{\xi}_{\tau}^{h}$ :

$$
\left\|\boldsymbol{u}^{h}\right\| \leq \alpha, \quad\left\|\boldsymbol{\xi}_{\tau}^{h}\right\|_{L^{2}\left(\Gamma_{3}\right)^{d}} \leq \beta+\delta\left\|\boldsymbol{u}^{h}\right\| .
$$

Since $V^{h} \subset V$, we observe that for each $\boldsymbol{v}^{h} \in V^{h}$ both (24) and (34) hold. We obtain

$$
\begin{aligned}
\langle B(\boldsymbol{u} & \left.\left.-\boldsymbol{u}^{h}\right), \boldsymbol{v}^{h}\right\rangle-\int_{\Gamma_{3}}\left(\boldsymbol{\xi}_{\tau}(x)\right. \\
& \left.-\boldsymbol{\xi}_{\tau}^{h}(x)\right) \cdot \boldsymbol{v}_{\tau}^{h}(x) \mathrm{d} \Gamma=0, \quad \forall \boldsymbol{v}^{h} \in V^{h} .
\end{aligned}
$$

By Lemma 2 we have

$$
m\left\|\boldsymbol{u}-\boldsymbol{u}^{h}\right\|^{2} \leq\left\langle B\left(\boldsymbol{u}-\boldsymbol{u}^{h}\right), \boldsymbol{u}-\boldsymbol{u}^{h}\right\rangle,
$$

and by the condition $H\left(j_{s}\right)(\mathrm{d})$ and $(20)$, after integration over $\Gamma_{3}$ we get

$$
\begin{aligned}
- & S \lambda C_{0}^{2}\left\|\boldsymbol{u}-\boldsymbol{u}^{h}\right\|^{2} \\
\leq- & \int_{\Gamma_{3}}\left(\boldsymbol{\xi}_{\tau}(x)-\boldsymbol{\xi}_{\tau}^{h}(x)\right) \cdot\left(\boldsymbol{u}_{\tau}(x)-\boldsymbol{u}_{\tau}^{h}(x)\right) \mathrm{d} \Gamma .
\end{aligned}
$$

From (41) and (42) we obtain for all $\boldsymbol{v}^{h} \in V^{h}$

$$
\begin{aligned}
(m- & \left.S \lambda C_{0}^{2}\right)\left\|\boldsymbol{u}-\boldsymbol{u}^{h}\right\|^{2} \\
\leq & \left\langle B\left(\boldsymbol{u}-\boldsymbol{u}^{h}\right), \boldsymbol{u}-\boldsymbol{u}^{h}\right\rangle \\
& -\int_{\Gamma_{3}}\left(\boldsymbol{\xi}_{\tau}(x)-\boldsymbol{\xi}_{\tau}^{h}(x)\right) \cdot\left(\boldsymbol{u}_{\tau}(x)-\boldsymbol{u}_{\tau}^{h}(x)\right) \mathrm{d} \Gamma \\
= & \left\langle B\left(\boldsymbol{u}-\boldsymbol{u}^{h}\right), \boldsymbol{u}-\boldsymbol{v}^{h}\right\rangle \\
& -\int_{\Gamma_{3}}\left(\boldsymbol{\xi}_{\tau}(x)-\boldsymbol{\xi}_{\tau}^{h}(x)\right) \cdot\left(\boldsymbol{u}_{\tau}(x)-\boldsymbol{v}_{\tau}^{h}(x)\right) \mathrm{d} \Gamma \\
\leq & \|B\|_{\mathcal{L}\left(V, V^{*}\right)}\left(\|\boldsymbol{u}\|+\left\|\boldsymbol{u}^{h}\right\|\right)\left\|\boldsymbol{u}-\boldsymbol{v}^{h}\right\| \\
& +\left(\left\|\boldsymbol{\xi}_{\tau}\right\|_{L^{2}\left(\Gamma_{3}\right)^{d}}+\left\|\boldsymbol{\xi}_{\tau}^{h}\right\|_{L^{2}\left(\Gamma_{3}\right)^{d}}\right) C_{0}\left\|\boldsymbol{u}-\boldsymbol{v}^{h}\right\| .
\end{aligned}
$$

The equality in 43) is a consequence of the application of (40) with $\boldsymbol{v}^{h}=\boldsymbol{u}^{h}$. Combining (37)-39) and (43), we obtain for all $\boldsymbol{v}^{h} \in V^{h}$

$$
\begin{aligned}
& \left(m-S \lambda C_{0}^{2}\right)\left\|\boldsymbol{u}-\boldsymbol{u}^{h}\right\|^{2} \\
& \quad \leq\|B\|_{\mathcal{L}\left(V, V^{*}\right)} 2 \alpha\left\|\boldsymbol{u}-\boldsymbol{v}^{h}\right\|+\kappa\left\|\boldsymbol{u}-\boldsymbol{v}^{h}\right\|
\end{aligned}
$$

where the constant $\kappa>0$ depends only on the data of the problem. Rearranging the last inequality and using the fact that $v^{h} \in V^{h}$ is arbitrary, we conclude the proof.

Remark 5. Theorem 3 means that the error of the Galerkin method has the order $O\left(\sqrt{\inf \boldsymbol{v}^{h} \in V^{h}\left\|\boldsymbol{u}-\boldsymbol{v}^{h}\right\|}\right)$. 
Remark 6. In the above case an estimation of the error between $\xi$ and $\xi^{h}$ is an open problem. To obtain such estimates, a discrete Babuska-Brezzi inf-sup condition is used. Such a condition requires the discretization of the contact boundary stress (cf. Hild and Renard, 2007).

\section{Nonconvex proximal bundle method}

In this section we use a numerical strategy of nonconvex and nonsmooth optimization known as the proximal bundle method to solve the Galerkin problem $\mathcal{P}_{V}^{h}$. To that end, we first need to reformulate it as a minimization problem in $\mathbb{R}^{n}$.

Let us consider a basis $\mathbb{B}=\left\{\boldsymbol{v}_{1}^{h}, \ldots, \boldsymbol{v}_{N}^{h}\right\} \subset V^{h}$ of the space $V^{h}$, where $N=\operatorname{dim} V^{h}$, and define the function $h: \mathbb{R}^{N} \rightarrow \mathbb{R}$ by

$$
h(\alpha)=\int_{\Gamma_{3}} j_{s}\left(\sum_{j=1}^{N} \alpha_{j} \boldsymbol{v}_{j}^{h}(x)\right) \mathrm{d} \Gamma, \quad \forall \alpha \in \mathbb{R}^{N} .
$$

We observe (cf. Clarke, 1983, Theorems 2.3.10 and 2.7.2) that if $\eta \in \partial h(\alpha)$ then there exists $\boldsymbol{\xi}_{\tau}^{h} \in L^{2}\left(\Gamma_{3}\right)^{d}$ such that $-\boldsymbol{\xi}_{\tau}^{h}(x) \in \partial j_{s}\left(\sum_{j=1}^{N} \alpha_{j} \boldsymbol{v}_{j}^{h}(x)\right)$ a.e. on $\Gamma_{3}$ and

$$
\begin{aligned}
& \langle\eta, \zeta\rangle_{\mathbb{R}^{N} \times \mathbb{R}^{N}} \\
& =\int_{\Gamma_{3}}-\boldsymbol{\xi}_{\tau}^{h}(x) \cdot\left(\sum_{j=1}^{N} \zeta_{j} \boldsymbol{v}_{j}^{h}(x)\right) \mathrm{d} \Gamma, \quad \forall \zeta \in \mathbb{R}^{N} .
\end{aligned}
$$

From this observation we conclude that Problem $\mathcal{P}_{V}^{h}$ can be replaced by the following.

Problem 5. $\left(\mathcal{P}_{\mathbb{R}^{N}}^{h}\right)$ Find $\alpha \in \mathbb{R}^{N}$ such that

$$
\mathcal{F}-\alpha^{T} \mathcal{B} \in \partial h(\alpha),
$$

where

$$
\begin{aligned}
\mathcal{F} & =\left[F_{i}\right]_{i=1}^{N}, & F_{i} & =\left\langle f, \boldsymbol{v}_{i}^{h}\right\rangle, \\
\mathcal{B} & =\left[\mathrm{B}_{i j}\right]_{i j=1}^{N}, & \mathrm{~B}_{i j} & =\left\langle B \boldsymbol{v}_{i}^{h}, \boldsymbol{v}_{j}^{h}\right\rangle .
\end{aligned}
$$

In fact, if $\bar{\alpha} \in \mathbb{R}^{N}$ satisfies 477, then $\bar{u}=\sum_{j=1}^{N} \bar{\alpha}_{j} \boldsymbol{v}_{j}^{h}$ solves Problem $\mathcal{P}_{V}^{h}$. By Proposition 2.3.3 of Clarke (1983), the inclusion (47) is equivalent to the following:

$$
0 \in \partial H(\alpha)
$$

where

$$
H(\alpha)=\frac{1}{2} \alpha^{T} \mathcal{B} \alpha-\mathcal{F}^{T} \alpha+h(\alpha)
$$

In the sequel we will use the fact that if $\alpha \in \mathbb{R}^{N}$ is a local minimizer (or maximizer) of the functional $H$ then it satisfies (48) (cf. Clarke, 1983, Proposition 2.3.2).
Remark 7. If the assumptions $H(\mathcal{E})(\mathrm{a})-(\mathrm{c}), H(f)$, $H(\mu)(\mathrm{a})-(\mathrm{d})$ as well as (28) hold, then the functional $H$ is convex and convex programming algorithms can be used for its minimization. If we omit $H(\mu)(\mathrm{d})$ and (28), then the solution is not necessarily unique and there is no error estimate provided by Theorem 3. only the strong convergence of a subsequence of Galerkin solutions to the exact solution can be proved (see Haslinger et al., 1999, Theorem 3.4). The nonconvex PBM, however, remains still valid in such a case.

Now we formulate the lemma on the existence of a global minimizer of $H$.

Lemma 4. Under the assumptions $H(\mathcal{E})(a)-(c), H(f)$ and $H(\mu)(a)-(c)$, the functional $H$ defined in (48) attains a global minimum.

Proof. It is enough to verify that the functional $H$ is proper, lower semicontinuous and coercive. The fact that $H$ is proper is obvious and the lower semi-continuity follows from the fact that $h$ is locally Lipschitz. For coercivity we need to estimate $H(\alpha)$ from below. Let $\alpha \in$ $\mathbb{R}^{N}$ and $\boldsymbol{v}=\sum_{i=1}^{N} \alpha_{i} \boldsymbol{v}_{i}^{h}$. Then by means of Lemma 2 and the Lebourg mean-value Theorem (see Clarke, 1983, Theorem 2.3.7) we obtain

$$
\begin{aligned}
H(\alpha)= & \frac{1}{2}\langle B \boldsymbol{v}, \boldsymbol{v}\rangle-\langle\boldsymbol{f}, \boldsymbol{v}\rangle+\int_{\Gamma_{3}} j_{s}\left(\boldsymbol{v}_{\tau}(x)\right)-j_{s}(0) \mathrm{d} \Gamma \\
& +\int_{\Gamma_{3}} j_{s}(0) \mathrm{d} \Gamma \\
\geq & \frac{1}{2} m\|\boldsymbol{v}\|^{2}-\|\boldsymbol{f}\|_{V^{*}}\|\boldsymbol{v}\| \\
& -\int_{\Gamma_{3}} \eta(x) \cdot\left(-\boldsymbol{v}_{\tau}(x)\right) d \Gamma+j_{s}(0) \operatorname{meas}\left(\Gamma_{3}\right),
\end{aligned}
$$

where $\eta(x) \in \partial j_{s}(\theta(x) \boldsymbol{v}(x))$ a.e. on $\Gamma_{3}$ with $\theta(x) \in$ $(0,1)$. Furthermore, using the conditions $H(j)(\mathrm{b})-(\mathrm{c})$, Corollary 1 and the Cauchy formula with $\varepsilon$, we get

$$
\begin{aligned}
& \int_{\Gamma_{3}} \eta(x) \cdot\left(-\boldsymbol{v}_{\tau}(x)\right) \mathrm{d} \Gamma \\
& =\int_{\Gamma_{3}} \frac{1}{\theta(x)} \eta(x) \cdot\left(-\theta(x) \boldsymbol{v}_{\tau}(x)\right) \mathrm{d} \Gamma \\
& \leq \int_{\Gamma_{3}} \frac{1}{\theta(x)} j_{s}^{0}\left(\theta(x) \boldsymbol{v}_{\tau}(x)\right. \\
& \left.\quad-\theta(x) \boldsymbol{v}_{\tau}(x)\right) \mathrm{d} \Gamma \\
& \leq \int_{\Gamma_{3}} S(c+d)\left|\boldsymbol{v}_{\tau}(x)\right| \mathrm{d} \Gamma \\
& \leq \frac{S^{2}(c+d)^{2} m\left(\Gamma_{3}\right)}{2 \varepsilon}-\frac{\varepsilon}{2} C_{0}^{2}\|\boldsymbol{v}\|^{2}, \quad \forall \varepsilon>0 .
\end{aligned}
$$

Using (49), 50 with $\varepsilon<m / C_{0}^{2}$ and the fact that the expression $\rho(\alpha)=\left\|\sum_{i=1}^{N} \alpha_{i} \boldsymbol{v}_{i}^{h}\right\|$ is a well defined norm on $\mathbb{R}^{N}$, we conclude that $H$ is coercive. 
Now we turn to the formulation of the proximal bundle algorithm for nonconvex optimization used to minimize the function $H$. Its scheme is given as Algorithm 1. We refer to the works of Mäkelä (1990; 2001), Mäkelä et al. (1999) as well as Haslinger et al. (1999) and the references therein for details.

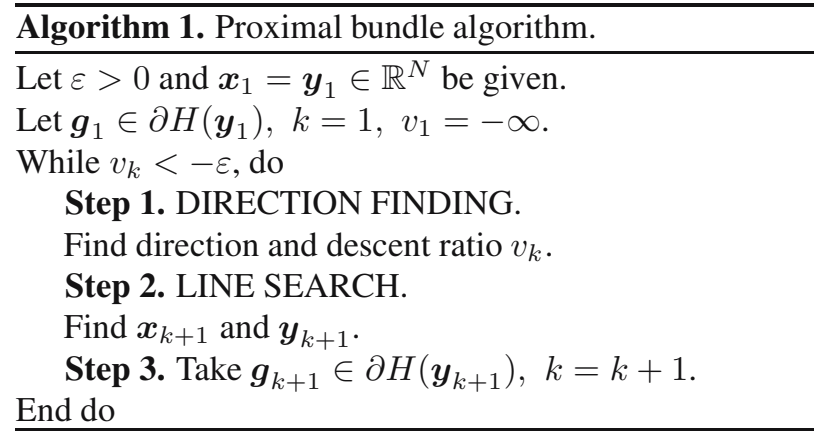

The algorithm constructs sequences $\left(\boldsymbol{x}_{k}\right)$ and $\left(\boldsymbol{y}_{k}\right)$. The starting point $\boldsymbol{x}_{1}$ is obtained as a solution to a frictionless problem, although it can be chosen arbitrarily. The sequence $\left(\boldsymbol{x}_{k}\right)$ is supposed to converge to a local minimum of $H$, while the auxiliary sequence $\left(\boldsymbol{y}_{k}\right)$ is used to construct the function $\hat{H}_{k}$, being a piecewise linear approximation of $H$. The search direction $\boldsymbol{d}_{k}$ is obtained as a solution to the problem

$$
\operatorname{minimize} \hat{H}_{k}\left(\boldsymbol{x}_{k}+\boldsymbol{d}\right)+\frac{1}{2} u_{k} \boldsymbol{d}^{T} \boldsymbol{d}, \quad \text { over } \boldsymbol{d} \in \mathbb{R}^{N}
$$

where the function $\hat{H}_{k}$ is defined as in the formulas (4), (5), (7), (8) by Mäkelä et al. (1999) and $u_{k}$ is an arbitrarily chosen weight. The problem (51) is a smooth quadratic programming problem with linear inequality constraints, which can be solved by standard quadratic optimization techniques. The value $v_{k}$ is the predicted amount of descent given by

$$
v_{k}=\hat{H}_{k}\left(\boldsymbol{x}_{k}+\boldsymbol{d}_{k}\right)-H\left(\boldsymbol{x}_{k}\right)
$$

The point $\boldsymbol{x}_{k+1}$ is obtained in the line search procedure as $\boldsymbol{x}_{k}+t_{L}^{k} \boldsymbol{d}_{k}$, where $t_{L}^{k} \in[0,1]$ is the largest number such that $H\left(\boldsymbol{x}_{k+1}\right) \leq H\left(\boldsymbol{x}_{k}\right)+m_{L} t_{L}^{k} v_{k}$ with $m_{L} \in(0,1 / 2)$ being an arbitrary parameter. Depending on the fact whether $t_{L}^{k}$ is greater or less than the arbitrary parameter $\bar{t} \in(0,1]$, either $\boldsymbol{y}_{k+1}=\boldsymbol{x}_{k+1}$ (the so-called long step) or $\boldsymbol{y}_{k+1}=\boldsymbol{x}_{k}+t_{R}^{k} \boldsymbol{d}_{k}$ with $t_{R}^{k}>t_{L}^{k}$ is given according to the formula (12) by Mäkelä et al. (1999) (the so-called short step). The idea of the short step is that, since we are in the vicinity of the nonsmoothness of $H$, its value is not decreasing significantly in the descent direction $\boldsymbol{d}_{k}$. Then we enrich the piecewise linear approximation by taking $\boldsymbol{y}_{k+1}$ on the other side of the nonsmoothness.

The Schur complement method is used for the compression of unknowns (see Haslinger et al., 1999,
Chapter 6.1), while for the solution of the problem (51) we use the LIBQP library (see Franc, 2011). For calculation of values of $h(\alpha)$ and an element of $\partial h(\alpha)$, the trapezoidal quadrature rule was used.

\section{Solution based on a sequence of convex programming problems}

The numerical strategy presented in this section is based on a sequence of convex programming problems; more details can be found in the works of Mistakidis and Panagiotopulos (1997; 1998). This approach is implemented by using an iterative procedure in which for each iteration the friction coefficient $\mu$ is fixed to a given function depending on the tangential displacement solution $\boldsymbol{u}_{\tau}$ found in the previous iteration. Then, the nonsmooth convex problems arising during the iterative process can be solved by classical numerical methods. In the following, we consider the discrete space $Y_{\tau}^{h} \subset$ $L^{2}\left(\Gamma_{3}\right)^{d}$ related to the discretization of the friction density $\boldsymbol{\sigma}_{\tau}$. We also consider the boundary interpolation operator $\Pi_{h}: V_{h} \rightarrow Y_{\tau}^{h}$ (see the works of Khenous et al. (2006a; 2006b) for more details about the discretization spaces). The numerical solution of the nonsmooth nonconvex variational problem $\mathcal{P}_{V}^{h}$ is based on the iterative scheme given as Algorithm 2.

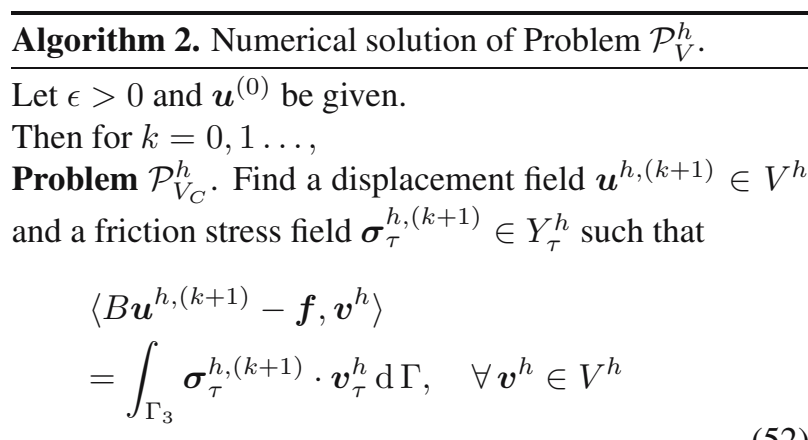

with

$$
-\boldsymbol{\sigma}_{\tau}^{h,(k+1)} \in \mu\left(\left|\Pi_{h} \boldsymbol{u}_{\tau}^{h,(k)}\right|\right) S \partial\left|\Pi_{h} \boldsymbol{u}_{\tau}^{h,(k+1)}\right| \text { on } \Gamma_{3}
$$

until $\left\|\boldsymbol{u}^{h,(k+1)}-\boldsymbol{u}^{h,(k)}\right\| \leq \epsilon\left\|\boldsymbol{u}^{h,(k)}\right\|$

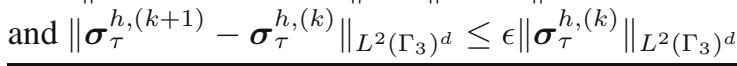

Here, $k$ represents the index of the iterative procedure and (53) is derived from (15) using (14). This numerical strategy leads to the solution of a nonsmooth convex problem $\mathcal{P}_{V_{C}}^{h}$ at each iteration $k$. See Fig.1 for a graphical representation of the iterative algorithm at a contact node.

In the rest of the section, to simplify the readability, we skip the dependence on the iteration index $k$. In Problem $\mathcal{P}_{V_{C}}^{h}$ the discrete stress $\sigma_{\tau}^{h}$ on the contact 


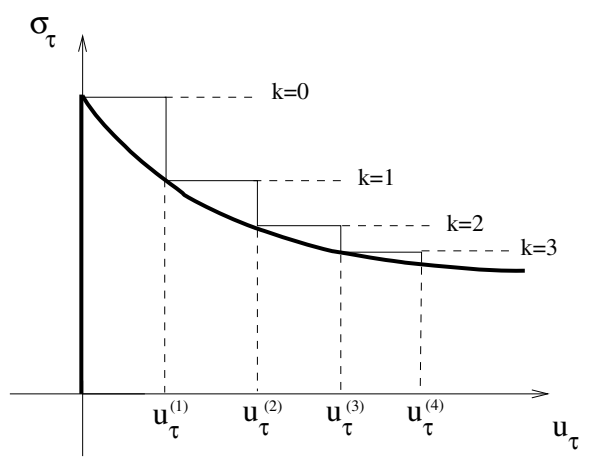

Fig. 1. Graphical description of the iterative algorithm at a contact node.

boundary $\Gamma_{3}$ can be viewed as a Lagrange stress multiplier.

The unique solvability of Problem $\mathcal{P}_{V_{C}}^{h}$ follows from arguments of variational inequalities and a fixed point theorem, similar to those used by Barboteu et al. (2002) as well as Han and Sofonea (2002). Moreover, the numerical analysis of this problem (including error estimates and convergence results) can be provided by extending the arguments already used by Barboteu et al. (2002) as well as Han and Sofonea (2002). Nevertheless, to keep this paper at a reasonable length, we skip this analysis and we pass in what follows to a brief description of the numerical algorithm used to solve Problem $\mathcal{P}_{V_{C}}^{h}$.

For the numerical treatment of the nonsmooth convex Problem $\mathcal{P}_{V_{C}}^{h}$ we use the augmented Lagrangean approach. To this end we consider additional fictitious nodes for the Lagrange multiplier in the initial mesh. The construction of these nodes depends on the contact element used for the geometrical discretization of the interface $\Gamma_{3}$. In the case of the numerical example presented in Section 7 the discretization is based on the "node-to-rigid" contact element, which is composed by one node of $\Gamma_{3}$ and one Lagrange multiplier node. This contact interface discretization is characterized by a finite dimensional subspace $H_{\Gamma_{3}}^{h} \subset Y_{\tau}^{h}$. Let $N_{\text {tot }}$ be the total number of nodes and denote by $\alpha^{i}$ the basis functions of the spaces $V^{h}$ for $i=1, \ldots, N_{t o t}$. Moreover, let $N_{\Gamma_{3}}$ represent the number of nodes on the interface $\Gamma_{3}$ and let $\mu^{i}$ be the shape functions of the finite element space $H_{\Gamma_{3}}^{h}$, for $i=1, \ldots, N_{\Gamma_{3}}$; thus,

$$
H_{\Gamma_{3}}^{h}=\left\{\gamma^{h} \in Y_{\tau}^{h}: \gamma^{h}=\sum_{i=1}^{N_{\Gamma_{3}}} \gamma^{i} \mu^{i}\right\}
$$

Usually, if a $P_{1}$ finite element method is used for the displacement, then a $P_{0}$ finite element method is considered for the multipliers (see Khenous et al., 2006a; 2006b). Then, the expression of functions $\boldsymbol{v}^{h} \in V^{h}$ and $\gamma^{h} \in H_{\Gamma_{3}}^{h}$ is given by

$$
\begin{aligned}
& \boldsymbol{v}^{h}=\sum_{i=1}^{N_{\text {tot }}} \boldsymbol{w}^{i} \alpha^{i}, \quad \forall \boldsymbol{v}^{h} \in V^{h}, \\
& \boldsymbol{\gamma}^{h}=\sum_{i=1}^{N_{\Gamma_{3}}} \gamma^{i} \mu^{i}, \quad \forall \boldsymbol{\gamma}^{h} \in H_{\Gamma_{3}}^{h},
\end{aligned}
$$

where $\boldsymbol{v}^{i}$ represent the values of the corresponding functions $\boldsymbol{v}^{h}$ at the $i$-th node of $\mathcal{T}^{h}$. Also, $\gamma^{i}$ denotes the values of the function $\gamma^{h}$ at the $i$-th node of the contact element discretization of the contact interface. More details about this discretization step can be found in the works of Alart and Curnier (1991), Khenous et al. (2006b), as well as Wriggers (2002).

The augmented Lagrangean approach we use shows that Problem $\mathcal{P}_{V_{c}}^{h}$ can be governed by a system of nonlinear equations of the following form.

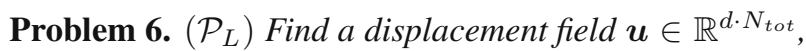
a stress multiplier field $\boldsymbol{\lambda} \in \mathbb{R}^{d \cdot N_{\Gamma_{3}}}$ such that

$$
\mathbf{R}(\boldsymbol{u}, \boldsymbol{\lambda})=\tilde{\mathbf{G}}(\boldsymbol{u})+\mathcal{F}(\boldsymbol{u}, \boldsymbol{\lambda})=\mathbf{0},
$$

where $\tilde{\mathbf{G}}$ and $\mathcal{F}$ are defined below.

A brief description of the notation used in this problem is the following.

First, the vectors, $\boldsymbol{u}$ and $\boldsymbol{\lambda}$ represent the displacement and the generalized Lagrange multiplier, respectively. They are defined by

$$
\boldsymbol{u}=\left\{\boldsymbol{u}^{i}\right\}_{i=1}^{N_{t o t}}, \quad \boldsymbol{\lambda}=\left\{\boldsymbol{\lambda}^{i}\right\}_{i=1}^{N_{\Gamma_{3}}},
$$

where $\boldsymbol{u}^{i}$ represents the value of the corresponding function $\boldsymbol{u}^{h}$ at the $i$-th node of $\mathcal{T}^{h}$. Also, $\lambda^{i}$ denotes the value of the corresponding function $\lambda^{h}$ at the $i$-th node of the contact element of the discretized contact interface.

In addition, the generalized elastic term $\tilde{\mathbf{G}}(\boldsymbol{u}) \in$ $\mathbb{R}^{d \cdot N_{t o t}} \times \mathbb{R}^{d \cdot N_{\Gamma_{3}}}$ is defined by $\tilde{\mathbf{G}}(\boldsymbol{u})=\left(\mathbf{G}(\boldsymbol{u}), \mathbf{0}_{d \cdot N_{\Gamma_{3}}}\right)$. Here $\mathbf{0}_{d \cdot N_{\Gamma_{3}}}$ is the zero element of $\mathbb{R}^{d \cdot N_{\Gamma_{3}}}$; also, $\mathbf{G}(\boldsymbol{u}) \in$ $\mathbb{R}^{d \cdot N_{\text {tot }}}$ denotes the elastic term, respectively, given by

$(\mathbf{G}(\boldsymbol{u}) \cdot(\boldsymbol{v}))_{\mathbb{R}^{d \times N_{\text {tot }}}}=\left\langle B \boldsymbol{u}^{h}-\boldsymbol{f}, \boldsymbol{v}^{h}\right\rangle, \quad \forall \boldsymbol{u}^{h}, \boldsymbol{v}^{h} \in V^{h}$.

Above, $\boldsymbol{u}$ and $\boldsymbol{v}$ represent the generalized vectors of coordinates $\boldsymbol{u}^{i}$ and $\boldsymbol{v}^{i}$ for $i=1, \ldots, N_{t o t}$, respectively, and note that the volume and surface efforts are contained in the term $\mathbf{G}(\boldsymbol{u})$.

The contact operator $\mathcal{F}(\boldsymbol{u}, \boldsymbol{\lambda})$, which allows dealing with the friction law, is given by

$$
\begin{aligned}
& (\mathcal{F}(\boldsymbol{u}, \boldsymbol{\lambda}) \cdot(\boldsymbol{v}, \boldsymbol{\gamma}))_{\mathbb{R}^{d \cdot N_{t o t}} \times \mathbb{R}^{d \cdot N_{\Gamma_{3}}}} \\
& =\int_{\Gamma_{3}} \nabla \boldsymbol{u} l_{\tau}^{r}\left(\boldsymbol{u}^{h}, \boldsymbol{\lambda}^{h}\right) \cdot \boldsymbol{v}^{h} \mathrm{~d} \Gamma \\
& +\int_{\Gamma_{3}} \nabla_{\boldsymbol{\lambda}} l_{\tau}^{r}\left(\boldsymbol{u}^{h}, \boldsymbol{\lambda}^{h}\right) \cdot \gamma^{h} \mathrm{~d} \Gamma, \\
& \forall \boldsymbol{u}, \boldsymbol{v} \in \mathbb{R}^{d \cdot N_{t o t}}, \forall \boldsymbol{\lambda}, \gamma \in \mathbb{R}^{d \cdot N_{\Gamma_{3}}}, \\
& \forall \boldsymbol{u}^{h}, \boldsymbol{v}^{h} \in V^{h}, \forall \boldsymbol{\lambda}^{h}, \gamma^{h} \in H_{\Gamma_{3}}^{h} .
\end{aligned}
$$


Here the Lagrangean multiplier $\boldsymbol{\lambda}$ and its virtual variable $\gamma$ represent the friction forces. Moreover, $l_{\tau}^{r}$ denotes the augmented Lagrangean functional given by

$$
\begin{aligned}
l_{\tau}^{r}\left(\boldsymbol{u}^{h}, \boldsymbol{\lambda}^{h}\right)= & \boldsymbol{u}_{\tau}^{h} \cdot \boldsymbol{\lambda}^{h}+\frac{r}{2}\left|\boldsymbol{u}_{\tau}^{h}\right|^{2} \\
& -\frac{1}{2 r}\left(\operatorname{dist}_{C[-\mu S]}\left\{\boldsymbol{\lambda}^{h}+r \boldsymbol{u}_{\tau}^{h}\right\}\right)^{2},
\end{aligned}
$$

where $r$ is a positive penalty coefficient and the Coulomb convex set $C[-\mu S]$ denotes the convex disk of radius $-\mu S$. For more details about the "quasi"-Lagrangean method, we refer the reader to the works of Alart and Curnier (1991) as well as Wriggers (2002).

The solution of the nonlinear system (54) is based on a linear iterative technique similar to that used in the Newton method. The latter permits to treat both variables $(\boldsymbol{u}, \boldsymbol{\lambda})$ simultaneously. Then, we consider the pair $\tilde{\boldsymbol{u}}=$ $(\boldsymbol{u}, \boldsymbol{\lambda})$ and the iterative scheme can be summarized as Algorithm 3.

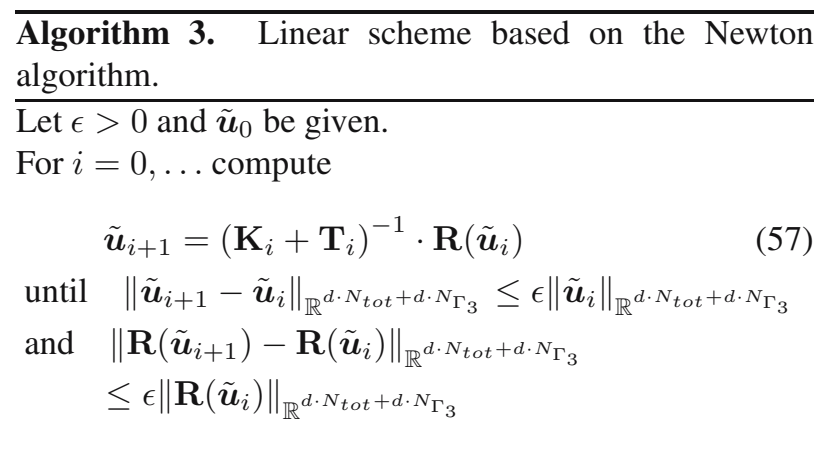

Here $i$ represents the Newton iteration index, $\mathbf{K}_{i}=$ $D_{\tilde{\boldsymbol{u}}} \mathbf{G}(\boldsymbol{u})$ denotes the usual elastic stiffness matrix and $\mathbf{T}_{i} \in \partial_{\tilde{\boldsymbol{u}}} \mathcal{F}\left(\tilde{\boldsymbol{u}}_{i}\right)$ is the frictional tangent matrix. $D_{\tilde{\boldsymbol{u}}} \mathbf{G}$ represents the differential of the functions $\mathbf{G}$ with respect to the variable $\tilde{\boldsymbol{u}} . \partial_{\tilde{\boldsymbol{u}}} \mathcal{F}\left(\tilde{\boldsymbol{u}}_{i}\right)$ represents the generalized Jacobian of $\mathcal{F}$ at $\tilde{\boldsymbol{u}}_{i}$. Usually $\tilde{\boldsymbol{u}}_{i}$ is in a region of linearity since the point set of nondifferentiability of the function $\mathcal{F}$ has null measure. Then $\mathbf{T}_{i}$ is reduced to a single classical Jacobian matrix. Each region of differentiability of the operator $\mathcal{F}$ corresponds to a friction state of each contact node of the discretization.

It is easy to see that 57 is equivalent to an algebraic linear system which can be solved by a conjugate gradient method with efficient preconditioners, used here to overcome the poor conditioning of the matrix due to the contact terms, see for instance, Alart et al. (1997). Details on computational contact mechanics, including algorithms similar to that described above, can be found in the monographs of Laursen (2002) and Wriggers (2002). Finally, recall that a similar numerical approach, in the study of contact problems with piezoelectric materials, is presented by Barboteu and Sofonea (2009). Details on these the classical algorithms can be found in the work of Alart and Curnier (1991), as well as Wriggers (2002).

\section{Numerical examples}

The main purpose of this section is to validate the numerical solution based on the PBM presented in Section 5 in comparison with the more standard method presented in Section 6. In order to do that, we consider an academic two-dimensional example of Problem $\mathcal{P}_{M}$ whose physical setting is depicted in Fig. 2 .

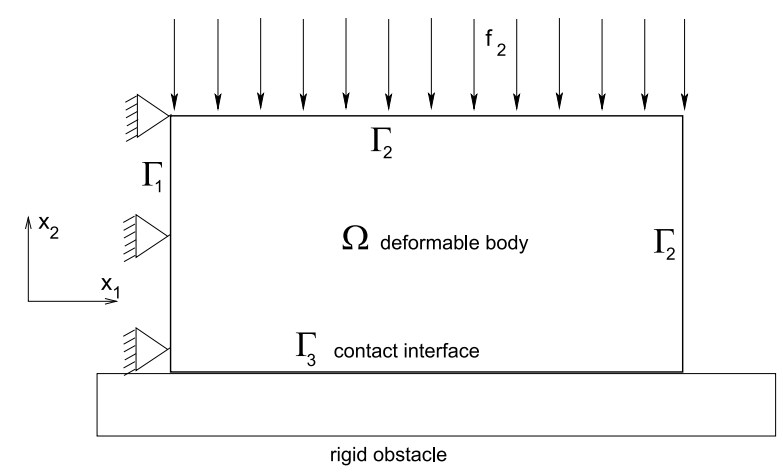

Fig. 2. Initial configuration of the two-dimensional example.

Let $\Omega=\left(0, L_{1}\right) \times\left(0, L_{2}\right) \subset \mathbb{R}^{d}, L_{1}, L_{2}>0$ be a rectangle with boundary $\Gamma$. We divide $\Gamma$ into three regions:

$$
\begin{aligned}
& \Gamma_{3}=\left[0, L_{1}\right] \times\{0\}, \\
& \Gamma_{1}=\{0\} \times\left[0, L_{2}\right], \\
& \Gamma_{2}=\Gamma \backslash\left(\Gamma_{1} \cup \Gamma_{3}\right) .
\end{aligned}
$$

There, we consider the domain $\Omega$ as the cross section of a three-dimensional linearly elastic body subjected to the action of tractions in such a way that a plane stress hypothesis is assumed. On the part $\Gamma_{1}=\{0\} \times\left[0, L_{2}\right]$ the body is clamped and therefore the displacement field vanishes there. Vertical tractions act on the part $\left[0, L_{1}\right] \times$ $\left\{L_{2}\right\}$ of the boundary, and the part $\left\{L_{1}\right\} \times\left[0, L_{2}\right]$ is traction free. Thus, $\Gamma_{2}=\left(\left\{L_{1}\right\} \times\left[0, L_{2}\right]\right) \cup\left(\left[0, L_{1}\right] \times\right.$ $\left.\left\{L_{2}\right\}\right)$. No body forces are assumed to act on the elastic body during the process. The body is in bilateral frictional contact with a rigid obstacle on the part $\Gamma_{3}=$ $\left[0, L_{1}\right] \times\{0\}$ of the boundary. The friction is modeled by a nonmonotone law in which the friction coefficient $\mu$ depends on the tangential displacement $\left|\boldsymbol{u}_{\tau}\right|$. Let us consider the following friction bound function $\mu: \mathbb{R}^{d} \rightarrow$ $\mathbb{R}$ :

$$
\mu\left(\left|\boldsymbol{u}_{\tau}\right|\right)=(a-b) \cdot e^{-\alpha\left|\boldsymbol{u}_{\tau}\right|}+b,
$$

with $a, b, \alpha>0, a \geq b$. See Fig. 3 for a representation of the friction bound function $\mu$ with the values $a=0.004$, $b=0.002$ and $\alpha=100$ used in the simulations. 


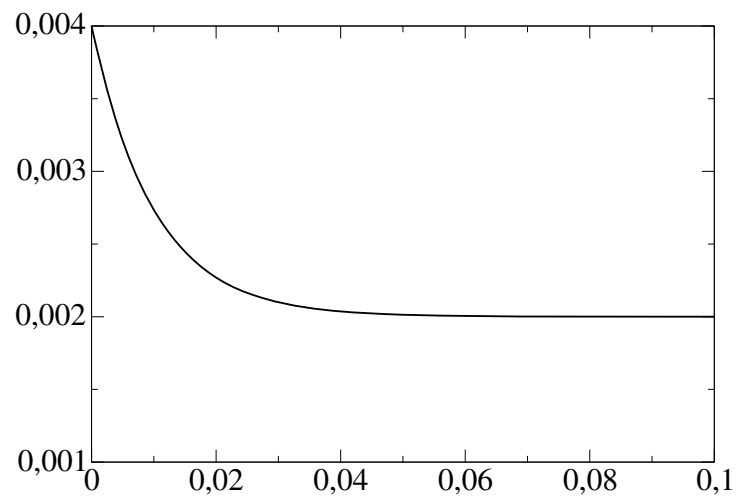

Fig. 3. Graph of the friction bound function $\mu$.

The elasticity tensor $\mathcal{E}$ satisfies

$$
(\mathcal{E} \boldsymbol{\tau})_{\alpha \beta}=\frac{E \kappa}{1-\kappa^{2}}\left(\tau_{11}+\tau_{22}\right) \delta_{\alpha \beta}+\frac{E}{1+\kappa} \tau_{\alpha \beta}
$$

with $1 \leq \alpha, \beta \leq 2$. $E$ is the Young modulus, $\kappa$ the Poisson ratio of the material and $\delta_{\alpha \beta}$ denotes the Kronecker symbol.

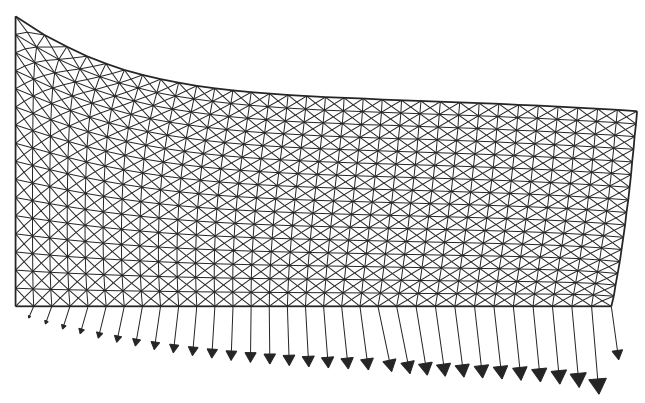

Fig. 4. Deformed mesh and contact interface forces on $\Gamma_{3}$.

For computation we used the following data:

$$
\begin{aligned}
& L_{1}=2 \mathrm{~m}, \quad L_{2}=1 \mathrm{~m}, \quad E=1 \mathrm{~N} / \mathrm{m}^{2}, \quad \kappa=0.3, \\
& \boldsymbol{f}_{0}=(0,0) \mathrm{N} / \mathrm{m}^{2}, \\
& \boldsymbol{f}_{2}=\left\{\begin{array}{rc}
(0,0) \mathrm{N} / \mathrm{m} & \text { on }\{2\} \times[0,1], \\
(0,-0.3) \mathrm{N} / \mathrm{m} & \text { on }[0,2] \times\{1\},
\end{array}\right. \\
& a=0.004, \quad b=0.002, \quad \alpha=100, \quad S=1 \mathrm{~N},
\end{aligned}
$$

stopping criterion: $\epsilon=10^{-6}$.

We used a structural mesh of triangles generated by taking the equidistant lines parallel to both axes and taking two diagonals in each of the obtained rectangles. In Fig. 4 the deformed configuration as well as the contact interface forces on $\Gamma_{3}$ are plotted.

Now, for both numerical approaches presented in Sections 5 and 6 the tangential stresses and the tangential displacements on $\Gamma_{3}$ are plotted respectively in Fig. 5, 8 In each of these figures, we plotted three curves corresponding to different values of the coefficients $a$ and $b$. The case $a=0.004$ and $b=0.002$ reflects the non-monotonicity of the friction law while the cases $a=$ $b=0.004$ and $a=b=0.002$ correspond to the classical monotone Coulomb law of dry friction. According to Figs. 5, 8 , we can see that the results obtained by the two numerical approaches are very similar with respect to the tangential stresses and the tangential displacements on the contact boundary $\Gamma_{3}$.

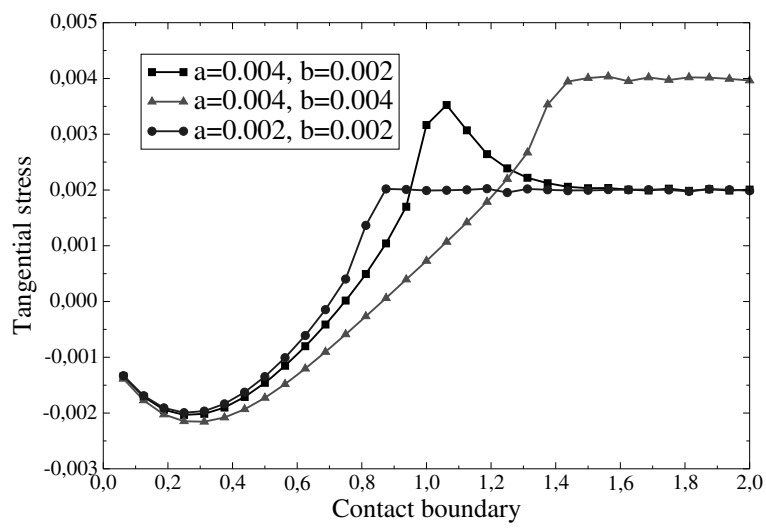

Fig. 5. Tangential stresses on $\Gamma_{3}$ for the PBM presented in Section 5

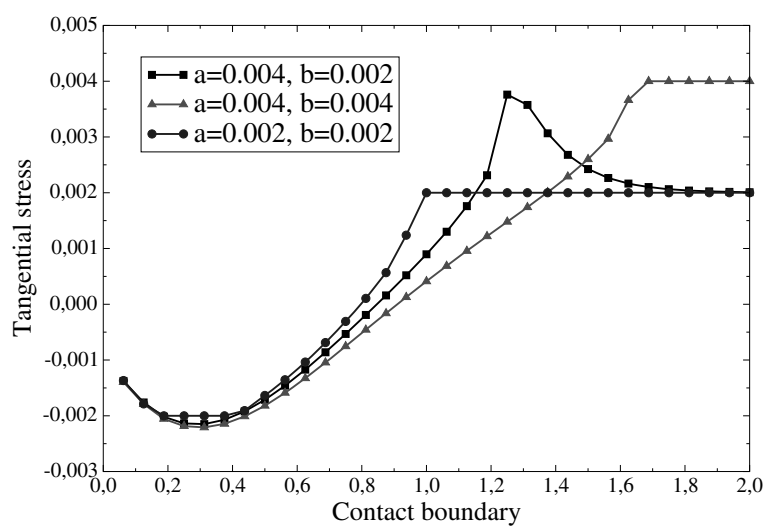

Fig. 6. Tangential stresses on $\Gamma_{3}$ for the algorithm presented in Section 6

In Fig. 9, we plotted the tangential stresses with respect to tangential displacements at the node of coordinates $(2.0,0.0)$ on $\Gamma_{3}$ during the iterations of the algorithm presented in Section 6 . According to Fig. 9 , we can find a nonmonotone behavior of the friction law characterized by the coefficients $a=0.004$ and $b=$ 0.002 . For $a=b=0.004$ and $a=b=0.002$, we recover a monotone friction behavior.

The details of the computations are the following. The problem is discretized in 2048 elastic finite elements and 32 contact elements. The total number of degrees of freedom is equal to 2210. For the algorithm presented in Section 6, the simulation needs 17 "convex" iterations to solve the nonconvex problems. The total number of 


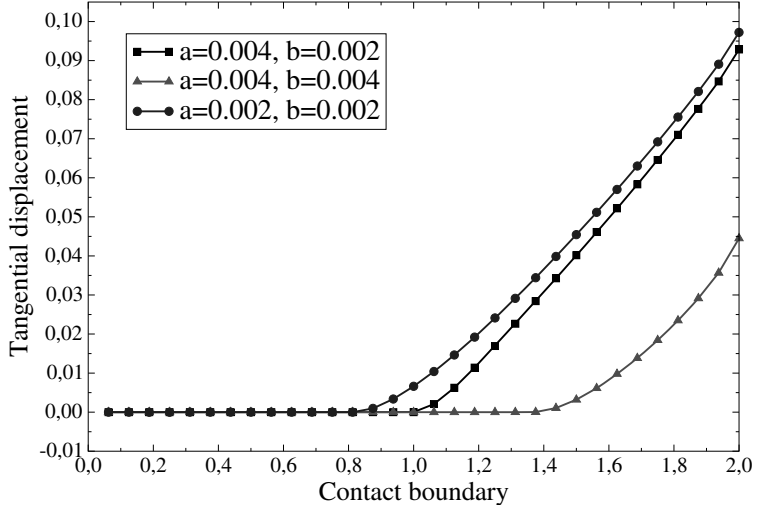

Fig. 7. Tangential displacements on $\Gamma_{3}$ for the PBM presented in Section 5

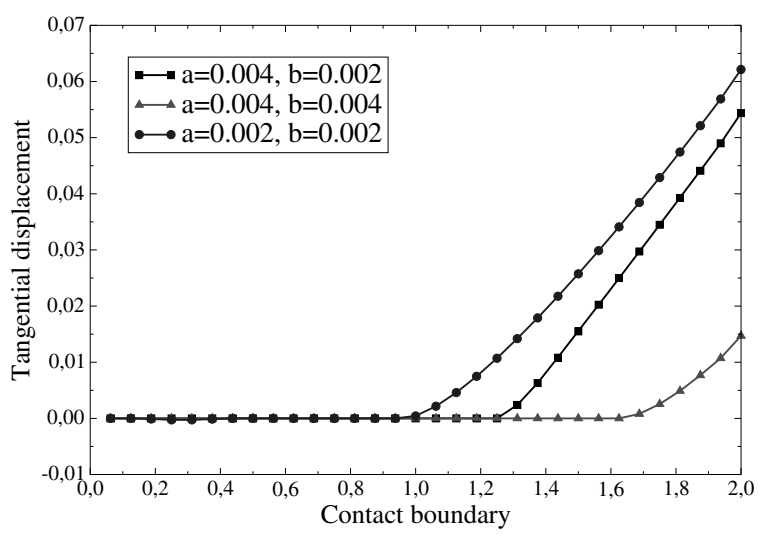

Fig. 8. Tangential displacements on $\Gamma_{3}$ for the algorithm presented in Section 6

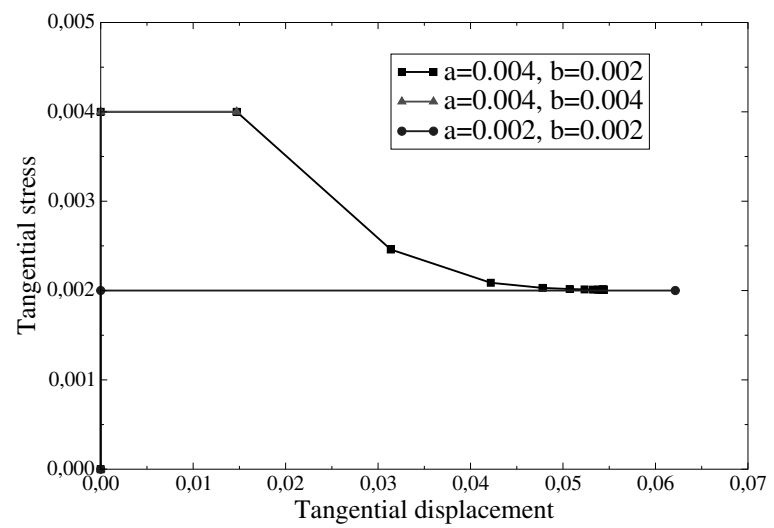

Fig. 9. Tangential stresses vs. tangential displacements at the node of coordinates $(2.0,0.0)$ on $\Gamma_{3}$ during the iterations of the algorithm presented in Section 6

Newton iterations for the solution of the nonsmooth and nonconvex problem is equal to 42 , whereas the number of iterations is equal to 8 for a nonsmooth convex problem characterized by $a=b=0.004$ or $a=b=0.002$. For the algorithm presented in Section 5, 541 iterations of the PBM loop were run for the case $a=b=0.002,598$ iterations for the case $a=b=0.004$ and 433 iterations for the case $a=0.004, b=0.002$. The parameters of the PBM used in computations (see Mäkelä et al., 1999) are the following: $\gamma=0.7, \bar{t}=0.2, m_{L}=0.2$, $m_{R}=0.6, u_{k}=0.01$. The trapezoidal formula for the numeric quadrature of the functional $h$ defined by (45) was used, where each boundary edge was divided into 5000 equidistant intervals. For the bisection in the PBM, 400 steps were used.

We can note that the number of the PBM iterations is almost constant for both convex and nonconvex problems. This is in contrast to the algorithm presented in Section 6 where the number of iterations strongly depends on the convexity of the problem. We also underline that for convex subproblems solved by means of the augmented Lagrangian method the friction condition is considered a constraint, and in consequence it is fixed in each step of the method. In contrast, the PBM does not force the friction condition to be satisfied in each iteration step since the friction term is a part of the objective functional. We do not make comparisons in terms of CPU time because the algorithmic steps of the two methods are very different and for some of these steps the performance can be optimized. However, we note that the method presented in Section 5 is faster than the PBM since the conditions of friction are exactly satisfied in each step of iteration.

As prospects, we plan to refine the comparison of these methods by considering, for instance, less academic numerical examples and problems with other tangential and normal contact laws.

\section{Acknowledgment}

This work was conducted within the Polonium project on Nonsmooth Analysis with Applications to Contact Mechanics under the contract no. 7817/R09/R10 between Jagiellonian University and the University of Perpignan. This research was also supported by a Marie Curie International Research Staff Exchange Scheme Fellowship within the 7th European Community Framework Programme under the grant agreement no. PIRSES-GA-2011-295118. The last two authors were also supported in part by the National Science Center of Poland under the grant no. N N201 604640, by an international project co-financed by the Ministry of Science and Higher Education of the Republic of Poland under the grant no. W111/7.PR/2012, and by the National Science Center of Poland under the Maestro Advanced Project no. DEC-2012/06/A/ST1/00262.

\section{References}

Alart, P., Barboteu, M. and Lebon, F. (1997). Solutions of frictional contact problems using an $E B E$ preconditioner, Computational Mechanics 30(4): 370-379. 
Alart, P. and Curnier, A. (1991). A mixed formulation for frictional contact problems prone to Newton like solution methods, Computer Methods in Applied Mechanics and Engineering 92(3): 353-375.

Baniotopoulos, C., Haslinger, J. and Moravkova, Z. (2005). Mathematical modeling of delamination and nonmonotone friction problems by hemivariational inequalities, Applications of Mathematics 50(1): 1-25.

Barboteu, M., Han, W. and Sofonea, M. (2002). Numerical analysis of a bilateral frictional contact problem for linearly elastic materials, IMA Journal of Numerical Analysis 22(3): 407-436.

Barboteu, M. and Sofonea, M. (2009). Analysis and numerical approach of a piezoelectric contact problem, Annals of the Academy of Romanian Scientists: Mathematics and Its Applications 1(1): 7-31.

Clarke, F.H. (1983). Optimization and Nonsmooth Analysis, Wiley Interscience, New York, NY.

Denkowski, Z., Migórski, S. and Papageorgiou, N.S. (2003). An Introduction to Nonlinear Analysis: Applications, Kluwer Academic/Plenum Publishers, Boston, MA/Dordrecht/London/New York, NY.

Duvaut, G. and Lions, J.L. (1976). Inequalities in Mechanics and Physics, Springer-Verlag, Berlin.

Franc, V. (2011). Library for quadratic programming, http://cmp. felk.cvut.cz/ xfrancv/libqp/html.

Han, W. and Sofonea, M. (2002). Quasistatic Contact Problems in Viscoelasticity and Viscoplasticity, American Mathematical Society, Providence, RI/International Press, Sommerville, MA.

Haslinger, J., Miettinen, M. and Panagiotopoulos, P.D. (1999). Finite Element Method for Hemivariational Inequalities. Theory, Methods and Applications, Kluwer Academic Publishers, Boston, MA/Dordrecht/London.

Hild, P. and Renard, Y. (2007). An error estimate for the Signorini problem with Coulomb friction approximated by finite elements, SIAM Journal of Numerical Analysis 45(5): 2012-2031.

Ionescu, I.R. and Nguyen, Q.L. (2002). Dynamic contact problems with slip-dependent friction in viscoelasticity, International Journal of Applied Mathematics and Computer Science 12(1): 71-80.

Ionescu, I.R., Nguyen, Q.L. and Wolf, S. (2003). Slip-dependent friction in dynamic elasticity, Nonlinear Analysis 53(3-4): 375-390.

Ionescu, I.R. and Paumier, J.C. (1996). On the contact problem with slip displacement dependent friction in elastostatics, International Journal of Engineering Sciences 34(4): 471-491.

Ionescu, I.R. and Sofonea, M. (1993). Functional and Numerical Methods in Viscoplasticity, Oxford University Press, Oxford.

Khenous, Y., Laborde, P. and Renard, Y. (2006a). On the discretization of contact problems in elastodynamics, in P. Wriggers and U. Nackenhorst (Eds.), Analysis and Simulation of Contact Problems, Lecture Notes in Applied and Computational Mechanics, Vol. 27, Springer, Berlin/Heidelberg, pp. 31-38.

Khenous, H.B., Pommier, J. and Renard, Y. (2006b). Hybrid discretization of the Signorini problem with coulomb friction. theoretical aspects and comparison of some numerical solvers, Applied Numerical Mathematics 56(2): 163-192.

Laursen, T. (2002). Computational Contact and Impact Mechanics, Springer, Berlin/Heidelberg.

Mäkelä, M.M. (1990). Nonsmooth Optimization, Theory and Applications with Applications to Optimal Control, Ph.D. thesis, University of Jyväskylä, Jyväskylä.

Mäkelä, M.M. (2001). Survey of bundle methods for nonsmooth optimization, Optimization Methods and Software 17(1): 1-29.

Mäkelä, M.M., Miettinen, M., Lukšan, L. and Vlček, J. (1999). Comparing nonsmooth nonconvex bundle methods in solving hemivariational inequalities, Journal of Global Optimization 14(2): 117-135.

Miettinen, M. (1995). On contact problems with nonmonotone contact conditions and their numerical solution, in M.H. Aliabadi and C. Alessandri (Eds.), Contact Mechanics II: Computational Techniques, Transactions on Engineering Sciences, Vol. 7, WIT Press, Southampton/Boston, MA, pp. 167-174.

Migórski, S. and Ochal, A. (2005). Hemivariational inequality for viscoelastic contact problem with slip-dependent friction, Nonlinear Analysis 61(1-2): 135-161.

Mistakidis, E.S. and Panagiotopulos, P.D. (1997). Numerical treatment of problems involving nonmonotone boundary or stress-strain laws, Computers \& Structures 64(1-4): 553-565.

Mistakidis, E.S. and Panagiotopulos, P.D. (1998). The search for substationary points in the unilateral contact problems with nonmonotone friction, Mathematical and Computer Modelling 28(4-8): 341-358.

Naniewicz, Z. and Panagiotopoulos, P.D. (1995). Mathematical Theory of Hemivariational Inequalities and Applications, Marcel Dekker, Inc., New York, NY/Basel/Hong Kong.

Nečas, J. and Hlavaček, I. (1981). Mathematical Theory of Elastic and Elastoplastic Bodies: An Introduction, Elsevier, Amsterdam.

Panagiotopoulos, P.D. (1985). Inequality Problems in Mechanics and Applications, Birkhauser, Basel.

Rabinowicz, E. (1951). The nature of the static and kinetic coefficients of friction, Journal of Applied Physics 22(11): 1373-1379.

Shillor, M., Sofonea, M. and Telega, J.J. (2004). Models and Analysis of Quasistatic Contact, Springer, Berlin.

Tzaferopoulos, M.A., Mistakidis, E.S., Bisbos, C.D. and Panagiotopulos, P.D. (1995). Comparison of two methods for the solution of a class of nonconvex energy problems using convex minimization algorithms, Computers \& Structures 57(6): 959-971. 
Wriggers, P. (2002). Computational Contact Mechanics, Wiley, Chichester.

Mikaël Barboteu received a Ph.D. degree in mechanics and applied mathematics from the Montpellier University of Sciences and Technologies in 1999. Since 2000 he has been a professor of applied mathematics in the Laboratory of Applied Mathematics and Physics (LAMPS) at the University of Perpignan Via Domitia. In 2006, he obtained the habilitation à diriger les recherches degree in applied and computational mathematics. His research interests include nonlinear and nonsmooth problems in mechanics (in particular, contact problems), variational and numerical analysis of PDEs, and computational mathematics.

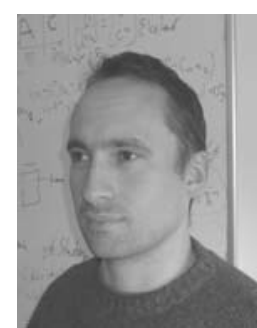

Krzysztof Bartosz received a Ph.D. degree in mathematics from Jagiellonian University in 2007. Since 2007 he has been first an assistant professor and then an associate professor in the Institute of Computer Science at the same university. His research interests include partial differential equations, variational and hemivariational inequalities, numerical methods, and mathematical modelling of contact problems in mechanics.

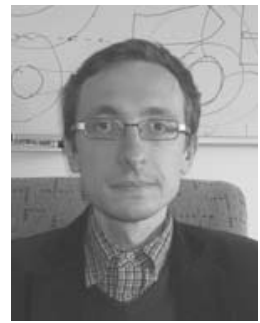

Piotr Kalita received a Ph.D. degree in computer science from Jagiellonian University in Kraków in 2005. Since 2008 he has been an associate professor in the Institute of Computer Science at Faculty of Mathematics and Computer Science at the same university. His research interests include mathematical and numerical analysis of weak solutions of partial differential equations and inclusions, as well as mathematical modelling in mechanics and biology.

Received: 19 July 2012

Revised: 30 October 2012 